\title{
Longitudinal Change of Sonographic Ovarian Aspects and Endocrine Parameters in Irregular Cycles of Adolescence
}

\author{
STEFANO VENTUROLI, ELEONORA PORCU, RAFFAELLA FABBRI, VALERIA PLUCHINOTTA, \\ SAVERIO RUGGERI, SILVIA MACRELLI, ROBERTO PARADISI, AND CARLO FLAMIGNI \\ Institute of Reproductive Physiology and Pathology [S.V., E.P., R.F., S.M., R.P., C.F.], Chair of Prenatal \\ Physiology and Pathology [V.P.], University of Bologna, Bologna, Italy, and G. B. Morgagni Hospital \\ Laboratories [S.R.], Forli, Italy
} \begin{abstract}
\begin{tabular}{|l} 
We longitudinally studied clinical endocrine and ultrasound \\
parameters of the ovaries in 73 healthy adolescents having
\end{tabular} persistent menstrual irregularities. After the first examination, they were reexamined after a variable period ranging from 2 to 7 $y$. During the first examination, three basic features of the ovaries were observed: homogeneous (36\%), multifollicular (23\%), and polycystic $(41 \%)$. Polycystic ovaries were most frequent, and they generally exceeded the normal adult range. During the last examination, in the entire group of irregular adolescents, homogeneous ovaries decreased $(-14 \%)$, polycystic ovaries increased $(+18 \%)$, and a further higher number of subjects exceeded the normal adult range $(+10 \%)$. The subjects with enlarged ovaries had the highest values of $\mathrm{LH}$, testosterone, and androstenedione. Fourteen subjects out of 46 (30\%), with normal ovarian volume in the first examination, registered an ovarian enlargement in the last examination, exceeding the normal range. Moreover, a change from the homogeneous or multifollicular structure to the polycystic one was observed. Twenty-one subjects out of 27 (78\%) with enlarged ovaries in the first examination confirmed the high ovarian volume and the unchanged structure in the last examination, whereas six subjects $(22 \%)$ showed ovaries within
\end{abstract}

the normal adult range; the polycystic structure was substantially confirmed. These results indicate the following. 1) Homogeneous, multifollicular, and polycystic ovaries can usually be found in the postmenarcheal period. 2) Enlarged ovaries, polycystic structure, hyperandrogenemia, and high $\mathrm{LH}$ values are strongly linked, and they are frequent in irregular cycles even in the absence of signs of hyperandrogenism. These characteristics may all persist or in various aggregations become a permanent feature. 3) Only a few subjects may lose ovarian enlargement and show a change in the polycystic structure; however, they frequently maintain hyperandrogenemia. 4) During the postmenarcheal period, normal ovarian characteristics may suddenly change, and the ovaries may take on a polycystic structure and increase in volume. Moreover, some endocrine parameters may reach pathologic levels. (Pediatr Res 38: 974-980, 1995)
$\mathbf{E}_{\mathbf{1}}$, estrone
Abbreviations
$\mathbf{E}_{2}$, estradiol
PCOS, polycystic ovary syndrome

It is well known that the volume and the structure of the ovaries can change strikingly during puberty and adolescence until adult age is reached $(1,2)$. Sudden changes in this period of life often make it difficult to codify ovarian normality and to differentiate physiologic or pathologic condition of the ovaries.

It is a fact that normal volume or enlarged and inhomogeneous ovaries, variously defined as multifollicular (3), multicystic (4), megalocystic (5), and polycystic (2), are a frequent feature of the adolescent period. They are often considered as normal features, but clinical doubts still exist regarding their evolution and implications for the adult reproductive function, particularly when associated with irregular menstrual cycles $(3,4)$.

Received December 19, 1994; accepted July 6, 1995

Correspondence and reprint requests: Prof. Stefano Venturoli, Clinica Ostetrica e Ginecologica, Via Massarenti N.13, I-40138, Bologna, Italia.
Irregular menstrual cycles tend to persist in the years after menarche, reflecting a still developing cyclic function. Even though they do not necessarily indicate a pathologic condition, irregular cycles are frequently associated with endocrine patterns which resemble full-blown pathology, such as PCOS (4, $6,7)$.

Considering that very few cross-sectional studies exist (8), we performed this longitudinal study to clarify the outcome of the ovaries and define their related clinical and endocrine parameters in a group of adolescents with irregular cycles.

\section{METHODS}

Subjects and experimental design. This series included 73 young healthy girls, suffering from menstrual irregularity which had persisted for a variable period since menarche. 
Subjects with additional clinical findings, such as acne, obesity, hirsutism, and physical or psychologic disease were excluded from the study. Sixty-three subjects out of 73 had long cycles (over $35 \mathrm{~d}$ and under $3 \mathrm{mo}$ ), and 10 had short cycles (under $25 \mathrm{~d}$ ).

Each subject first came to the hospital in 1983 as an outpatient. Each subject at the moment of the first examination had a protocol consisting of clinical evaluation and including hirsutism score, blood samples for hormonal determinations, and ultrasound evaluation of the ovaries, and they had a successive reexamination after a variable period, ranging from 2 to $7 \mathrm{y}$. Each subject agreed not to use hormonal medications during the entire study period; on the occasions in which this occurred, the patient was dropped from the study. The purpose of the research was explained to each subject, and informed consent was obtained according to the Declaration of Helsinki.

Clinical data of the patients at the first and last examination are reported in Table 1 . All of the subjects at the first examination were between 11.7 and $19.8 \mathrm{y}$ of age in chronologic age and between 0.8 and $7.8 \mathrm{y}$ in gynecologic age (years after menarche). At the last examination they were between 13.8 and $24.6 \mathrm{y}$ in chronologic age, and between 2.1 and $14 \mathrm{y}$ in gynecologic age.

Adolescents with irregular cycles were also divided into two groups on the basis of their ovarian volume. Group 1 had ovarian volume within normal values and group 2 had ovarian volume exceeding normal adult range (adult range $=4.6-10.8$ $\left.\mathrm{cm}^{3}\right)(3,4)$.

Twenty healthy ovulatory adolescents with well established regular cycles from menarche were used as a control group. Endocrine and ultrasound data are reported in Table 1; mean chronologic age of control group was $15 \pm 1.2 \mathrm{y}$, mean gynecologic age was $2.7 \pm 1.3 \mathrm{y}$, and mean cycle length was $29 \pm 2 \mathrm{~d}$.

Ultrasound examination. Transabdominal ultrasound examination of the ovaries was performed during the early follicular phase of the cycle (d 1-7) and was carried out using a $3.5 \mathrm{MHz}$ transducer sector scanner (SRT L/S General Electric, Milwaukee, WI). The procedure was always performed by the same two operators who cyclically compared the results to assess the reproducibility of the measurements made.

Ovarian volume was calculated using the formula for a prolate ellipsoid as previously reported $(3,4)$. Two sets of volume measurements, made by each observer on the same occasion, were periodically regressed against each other. The correlation coefficient for the volume measurements ranged between 0.94 and 0.97 . The mean coefficient of variation between volume measurements was $9.1 \%$.

The echogenic texture of the ovaries was defined, according to the presence of echo-free areas, as follows: 1 ) homogeneous, if less than four cystic areas were imaged; 2) multifollicular, if multiple (4-10) cystic areas, 5-10 $\mathrm{mm}$ large and spread over the ovary, were present, and the stroma was of normal echogenicity; 3) polycystic, if there were multiple cysts (10 or more) $3-8 \mathrm{~mm}$ in diameter arranged peripherally or located in the inner part of the ovary. The presence of an echodense stroma should mean an increased amount of stroma, even though the increase (9), in our opinion, is prone to the varia-
Table 1. Clinical parameters and ovarian and endocrine data of adolescents with irregular cycles during the first and the last longitudinal examination compared with normal adolescents

\begin{tabular}{|c|c|c|c|}
\hline \multirow[b]{3}{*}{ Parameter } & \multicolumn{3}{|c|}{ Adolescents } \\
\hline & \multirow{2}{*}{$\begin{array}{l}\text { With } \\
\text { regular cycles }\end{array}$} & \multicolumn{2}{|c|}{ With irregular cycles } \\
\hline & & First control & Last control \\
\hline Number of subjects & 20 & 73 & 73 \\
\hline Height $(\mathrm{cm})$ & $157.4 \pm 9.3$ & $158.5 \pm 11.4$ & $161 \pm 6.6$ \\
\hline Weight $(\mathrm{kg})$ & $54.7 \pm 8.1$ & $54.9 \pm 10.3$ & $55.1 \pm 9.2$ \\
\hline Menarche (mo) & $147.6 \pm 14$ & $155 \pm 18$ & \\
\hline Cronological age (mo) & $180.4 \pm 14$ & $191.4 \pm 21.5^{E}$ & $222.2 \pm 28.5$ \\
\hline Gynecological age (mo) & $33.1 \pm 15.3$ & $36.6 \pm 23.9^{E}$ & $67.5 \pm 30.1$ \\
\hline Cycle length $(\mathrm{d})$ & $29 \pm 2$ & $44.1 \pm 21.2^{E}$ & $34.1 \pm 13.3$ \\
\hline $\begin{array}{l}\text { Frequency of luteinization, } \\
\quad n(\%)\end{array}$ & $20(100)$ & $34(47)$ & $50(68)$ \\
\hline Anovulation, $n(\%)$ & & $39(53)$ & $23(32)$ \\
\hline Ovarian volume $\left(\mathrm{cm}^{3}\right)^{*}$ & $6.9 \pm 2.4$ & $9.5 \pm 4.4^{b A}$ & $11.1 \pm 4.7^{h}$ \\
\hline Right ovary & & $9.9 \pm 4.7^{A}$ & $11.4 \pm 4.6$ \\
\hline Left ovary & & $9.1 \pm 4.2^{A}$ & $10.6 \pm 4.9$ \\
\hline \multicolumn{4}{|l|}{ Ovarian structure: } \\
\hline \multicolumn{4}{|l|}{ Right ovary, $n(\%)$} \\
\hline $\mathrm{H}$ & $14(70)$ & $26(36)$ & $16(22)$ \\
\hline $\mathrm{P}$ & $2(10)$ & $30(41)$ & $43(59)$ \\
\hline $\mathrm{M}$ & $4(20)$ & $17(23)$ & $14(19)$ \\
\hline \multicolumn{4}{|l|}{ Left ovary, $n(\%)$} \\
\hline $\mathrm{H}$ & & $29(39)$ & $13(18)$ \\
\hline $\mathrm{P}$ & & $32(43)$ & $45(59)$ \\
\hline M & & $13(18)$ & $15(20)$ \\
\hline FSH (IU/L) & $7.9 \pm 2.6$ & $8.1 \pm 2.8^{B}$ & $9.5 \pm 3.8$ \\
\hline LH (IU/L) & $6.7 \pm 3.9$ & $12.9 \pm 11.6^{a}$ & $15.4 \pm 9.8^{h}$ \\
\hline $\mathrm{E}_{1}(\mathrm{pmol} / \mathrm{L})$ & $96 \pm 44$ & $100 \pm 66$ & $89 \pm 59$ \\
\hline $\mathrm{E}_{2}(\mathrm{pmol} / \mathrm{L})$ & $88 \pm 37$ & $103 \pm 70$ & $99 \pm 66$ \\
\hline $\mathrm{E}_{2}(\mathrm{pmol} / \mathrm{L}) \dagger$ & $316 \pm 172$ & $209 \pm 110^{c D}$ & $321 \pm 198$ \\
\hline $\mathrm{P}(\mathrm{nmol} / \mathrm{L}) \dagger$ & $21.9 \pm 12.4$ & $7.9 \pm 11.4^{d B}$ & $13.9 \pm 13.0^{e}$ \\
\hline $\mathrm{T}(\mathrm{nmol} / \mathrm{L})$ & $0.90 \pm 0.38$ & $1.11 \pm 0.38^{a C}$ & $1.39 \pm 0.76^{f}$ \\
\hline $\mathrm{A}(\mathrm{nmol} / \mathrm{L})$ & $4.19 \pm 1.35$ & $5.68 \pm 2.18^{c B}$ & $6.69 \pm 2.70^{h}$ \\
\hline DHEAS $(\mu \mathrm{mol} / \mathrm{L})$ & $4.88 \pm 2.17$ & $5.69 \pm 2.44^{E}$ & $7.32 \pm 3.26^{g}$ \\
\hline
\end{tabular}

* Mean ovarian volume (right + left $) \pm \mathrm{SD} . \mathrm{H}$, homogeneous; $\mathrm{P}$, polycystic; M, multifollicular.

$\dagger$ Luteal phase. P, progesterone; T, testosterone; A, androstenedione; DHEAS, dehydroepiandrosterone sulfate.

${ }^{a} p<0.05$ control $v s$ first.

${ }^{b} p<0.01$ control $v s$ first.

${ }^{c} p<0.005$ control $v s$ first.

${ }^{d} p<0.001$ control $v$ f first.

${ }^{e} p<0.05$ last $v s$ control.

${ }^{f} p<0.01$ last $v$ s control.

${ }^{g} p<0.005$ last $v s$ control.

${ }^{h} p<0.001$ last $v s$ control.

${ }^{A} p<0.05$ first $v$ s last.

${ }^{B} p<0.02$ first $v s$ last.

${ }^{c} p<0.01$ first $v$ s last.

${ }^{D} p<0.005$ first $v$ s last.

${ }^{E} p<0.001$ first $v s$ last.

tions due to the subjective interpretation of the operator in transabdominal examinations. Transabdominal ultrasound examination was the only available method at the beginning of the study because of the young age of the patients. It was mandatory to perform the subsequent evaluation with the same probe to avoid any methodologic bias in the longitudinal study.

Sample collection. Blood samples were collected at $0800 \mathrm{~h}$, starting on the 4 th $d$ of the cycle and every $7 \mathrm{~d}$ throughout the menstrual cycle, until the next menstrual bleeding, in an attempt to accurately determine the incidence of ovulation and 
standardize the endocrine data. The hormonal determinations and data analysis were performed on the first sample (4th $d$ of the cycle), which was believed to be indicative of the early follicular phase, and on the sample falling within -10 and -4 $\mathrm{d}$ before the following menstrual bleeding, which was thought to be indicative of the premenstrual phase.

Assays. Plasma FSH, LH, $\mathrm{E}_{1}, \mathrm{E}_{2}$, progesterone, testosterone, androstenedione, and dehydroepiandrosterone sulfate concentrations were measured in duplicate using RIA techniques as previously described (8). The methods and the antisera of the assay did not change during the entire period of the study.

Intra- and interassay variability (coefficients of variation) and sensitivity of the methods (8), periodically controlled during the years of the study, did not significantly change, assuring that any variations were not due to the modification of the method. In each assay a serum pool at three different concentrations (low, intermediate, and high levels) was also analyzed, and another three samples at different levels were assayed during two following trials to check the reproducibility and the variability of the data.

On the basis of our previous experience (8), progesterone levels exceeding $2 \mathrm{ng} / \mathrm{mL}$ in the premenstrual phase of the cycle were assumed to signify an ovulatory cycle.

Statistics. Statistical analyses were performed by analysis of variance and paired and unpaired $t$ test, as appropriate. Results are expressed in mean $\pm S D$.

\section{RESULTS}

\section{First Examination}

Ultrasound data. Table 1 reports ovarian data observed at the first examination. No more than $6-7 \%$ of subjects had a right ovarian volume and structure different from the left one in the same examination, and the right ovary was generally used for classification and analysis of the data. Using the left ovary, the results did not substantially change. Mean ovarian volume (as mean of left and right ovaries) of subjects with menstrual irregularities was higher than that of eumenorrheic adolescents $\left(9.50 \pm 4.4\right.$ versus $\left.6.9 \pm 2.1 \mathrm{~cm}^{3}, p<0.02\right)$ and that of adults $\left(9.50 \pm 4.4\right.$ versus $\left.7.6 \pm 2.1 \mathrm{~cm}^{3}, p<0.02\right)$.

Thirty-six percent of the subjects showed homogeneous ovaries (right ovary): $23 \%$ were multifollicular ovaries, whereas the most frequently observed were polycystic ovaries $(41 \%)$. Some doubts existed in the classification of the different ovarian structures when echographic parameters tended to overlap, and a subjective opinion cannot always be excluded, but it did not happen frequently.

When we considered the ovaries with polycystic structure altogether, ovarian volume was significantly higher $(12.1 \pm$ $\left.4.4 \mathrm{~cm}^{3}\right)$ with respect to homogeneous $\left(7.2 \pm 3.0 \mathrm{~cm}^{3}, p<\right.$ $0.001)$ and multifollicular ovaries $\left(8.2 \pm 4.4 \mathrm{~cm}^{3}, p<0.001\right)$.

Forty-six subjects (63\%, right ovary) had an ovarian volume within the normal range (group 1), with a sharp dominance of homogeneous (57\%) and multifollicular ovaries (28\%), whereas very few polycystic ovaries were found $(15 \%)$ (Table 2, Fig. 1).

Twenty-seven subjects ( $37 \%$, as referred to right ovary) exceeded normal ovarian range (group 2); 23 (85\%) had polycystic structure, and only $4(15 \%)$ subjects out of 27 showed a multifollicular structure (Table 2, Fig. 2).

Only a slight difference was found to exist in ovarian volume when ovulatory cycles were separated from anovulatory cycles $\left(9.1 \pm 4.1\right.$ versus $\left.10.5 \pm 4.7 \mathrm{~cm}^{3}, p=\mathrm{NS}\right)$.

Endocrine and clinical data. The means of hormone values are reported in Table 1. The entire group of irregular subjects, compared with regular adolescents, showed higher values of LH, testosterone, and androstenedione. The ovulatory cycles (Table 1) were $47 \%$, and progesterone and $E_{2}$ values in the premenstrual phase were lower than in the controls. Tests in $28 \%$ of subjects for $\mathrm{LH}, 22 \%$ for testosterone, and $33 \%$ for androstenedione exceeded the normal adolescent range.

Comparing subjects with normal (group 1) and enlarged (group 2) ovaries (endocrine and clinical data are compared in Table 2), FSH, LH, $\mathrm{E}_{1}$, testosterone, and androstenedione values in the follicular phase were significantly higher in group 2 than in group 1. A lower percentage of ovulations was registered in group 2, whereas no differences were observed in $\mathrm{E}_{2}$ and $p$ values in the luteal phase. Group 2 had a higher percentage of subjects exceeding the normal range with regard to $\mathrm{LH}$ (40 versus $17 \%$ ), testosterone (29 versus $17 \%$ ), and androstenedione (44 versus $28 \%$ ).

\section{Last examination}

Ultrasound data. In the entire group of adolescents, the mean ovarian volume increased with respect to the first examination (Table 1). Percentages of the different kinds of structures also changed; the number of homogeneous ovaries decreased (right ovary, $-14 \%$ ). There was only a small change in the percentage of multifollicular ovaries, whereas we found a higher percentage of polycystic ovaries increasing from 41 to $59 \%$. The ovarian volumes of the polycystic, homogeneous, and multifollicular ovaries increased to $13.1 \pm 5.2,7.9 \pm 3.2$, $9.0 \pm 4.3 \mathrm{~cm}^{3}$, respectively.

Thirty-eight subjects $(52 \%$, that is $-8 \%$ with respect to the first examination of the right ovary) had an ovarian volume within the normal range (Table 2). An increase of inhomogeneous ovaries was registered: 14 ovaries were homogeneous, 12 ovaries were polycystic, and 12 multifollicular.

Thirty-five subjects $(47 \%$, that is $+10 \%$ with respect to the first examination of the right ovary) exceeded the normal range with regard to ovarian volume (Table 2); a higher percentage of polycystic ovaries was observed with respect to the first examination. Thirty-one were polycystic, two multifollicular, and two were homogeneous.

No differences in ovarian volume were observed when ovulatory cycles were separated from anovulatory cycles $(11.5 \pm$ 5.2 versus $11.6 \pm 6.5 \mathrm{~cm}^{3} ; p=\mathrm{NS}$ ), even though an increase of ovarian volume was registered with respect to the first examination both in ovulatory $(p<0.025)$ and anovulatory cycles $(p=\mathrm{NS})$.

Endocrine and clinical data. The mean values of FSH, LH, testosterone, androstenedione, and dehydroepiandrosterone sulfate in the follicular phase and $\mathrm{E}_{2}$ and progesterone in the luteal phase increased (Table 1) during the last examination, and they were at their highest levels with respect both to the 
Table 2. Clinical parameters, ovarian and endocrine data of subjects found with ovaries under $10.8 \mathrm{~cm}^{3}$ (group 1) and those found with ovaries exceeding $10.8 \mathrm{~cm}^{3}$ (group 2) in the first and last examinations (the same parameters are also reported for the subgroup of subjects with increased and normalized ovaries)

\begin{tabular}{|c|c|c|c|c|c|c|c|c|}
\hline \multirow[b]{2}{*}{ Parameter } & \multicolumn{2}{|c|}{ Group 1} & \multicolumn{2}{|c|}{ Group 2} & \multicolumn{2}{|c|}{ Increased } & \multicolumn{2}{|c|}{ Normalized } \\
\hline & First & Last & First & Last & First & Last & First & Last \\
\hline Subjects, $n(\%)$ & $46(63)$ & & $27(37)$ & & 14 & 14 & 6 & 6 \\
\hline Weight $(\mathrm{kg})$ & $55 \pm 10$ & & $55 \pm 11$ & & $54 \pm 12$ & $53 \pm 7$ & $55 \pm 14$ & $53 \pm 12$ \\
\hline Height $(\mathrm{cm})$ & $155 \pm 6$ & & $157 \pm 6$ & & $159 \pm 6$ & $161 \pm 5$ & $163 \pm 4$ & $165 \pm 5$ \\
\hline Menarche (mo) & $151 \pm 17^{A}$ & & $160 \pm 18$ & & $163 \pm 16$ & & $155 \pm 10$ & \\
\hline Cronological age (mo) & $190 \pm 23$ & & $194 \pm 18$ & & $201 \pm 19$ & $240 \pm 23^{c}$ & $189 \pm 24$ & $221 \pm 23$ \\
\hline Gynecological age (mo) & $37.2 \pm 23.3$ & & $35.6 \pm 25.3$ & & $38 \pm 28$ & $77 \pm 30^{c}$ & $37 \pm 25$ & $68 \pm 23$ \\
\hline Cycle length (d) & $34.5 \pm 16^{B}$ & & $46.8 \pm 24.5$ & & $48 \pm 12$ & $36 \pm 6^{c}$ & $59 \pm 20^{d}$ & $34 \pm 7$ \\
\hline Frequency of luteinization, $n(\%)$ & $26(56.5)$ & & $9(33)$ & & $7(50)$ & $10(71)$ & $2(33)$ & $2(33)$ \\
\hline Ovarian volume $\left(\mathrm{cm}^{3}\right)^{*}$ & $7.17 \pm 2.68$ & $8 \pm 3$ & $13.6 \pm 3.75$ & $14.5 \pm 4.7$ & $8.2 \pm 3^{b}$ & $13 \pm 3.4$ & $14.2 \pm 4.2^{d}$ & $9.7 \pm 1.9$ \\
\hline Right ovary & $6.98 \pm 2.2$ & $7.6 \pm 2.1$ & $15.1 \pm 3.1$ & $16 \pm 4.6$ & $7.9 \pm 2.1^{b}$ & $13.8 \pm 1.8$ & $15.1 \pm 2.5^{e}$ & $9.5 \pm 1.1$ \\
\hline Left ovary & $7.36 \pm 3.13$ & $8.4 \pm 3.9$ & $12.1 \pm 4.4$ & $13 \pm 4.9$ & $8.5 \pm 3.9^{a}$ & $12.2 \pm 5.1$ & $13.4 \pm 6$ & $9.9 \pm 2.8$ \\
\hline \multicolumn{9}{|l|}{ Ovarian structure:* } \\
\hline \multicolumn{9}{|l|}{ Right ovary, $n(\%)$} \\
\hline $\mathrm{H}$ & $26(57)$ & $14(36)$ & 0 & $2(6)$ & $7(50)$ & 0 & 0 & 0 \\
\hline $\mathrm{P}$ & $7(15)$ & $12(31.5)$ & $23(85)$ & $31(88)$ & $3(21)$ & $13(93)$ & $5(83)$ & $4(66)$ \\
\hline M & $13(28)$ & $12(31.5)$ & $4(15)$ & $2(6)$ & $4(29)$ & $1(7)$ & $1(17)$ & $2(34)$ \\
\hline \multicolumn{9}{|l|}{ Ovarian structure:* } \\
\hline \multicolumn{9}{|l|}{ Left ovary, $n(\%)$} \\
\hline $\mathrm{H}$ & $23(49)$ & $13(34)$ & 0 & $3(8)$ & $9(65)$ & $1(7)$ & 0 & 0 \\
\hline $\mathrm{P}$ & $10(21)$ & $11(29)$ & $22(81)$ & $28(81)$ & $3(21)$ & $21(86)$ & $5(83)$ & $4(66)$ \\
\hline M & $14(30)$ & $14(37)$ & $5(19)$ & $4(21)$ & $2(14)$ & $1(7)$ & $1(17)$ & $2(34)$ \\
\hline FSH (IU/L) & $8.6 \pm 3^{A}$ & & $7.2 \pm 2.4$ & & $9.2 \pm 3.5$ & $8.7 \pm 4.7$ & $9.1 \pm 2.6$ & $10.3 \pm 4.8$ \\
\hline $\mathrm{LH}(\mathrm{IU} / \mathrm{L})$ & $10.5 \pm 7.1^{A}$ & & $15.2 \pm 11$ & & $11.5 \pm 8$ & $14.6 \pm 10.4$ & $13.5 \pm 12$ & $11 \pm 7$ \\
\hline $\mathrm{E}_{1}(\mathrm{pmol} / \mathrm{L})$ & $85 \pm 51^{B}$ & & $122 \pm 81$ & & $92 \pm 48$ & $103 \pm 81$ & $137 \pm 103$ & $96 \pm 48$ \\
\hline $\mathrm{E}_{2}(\mathrm{pmol} / \mathrm{L})$ & $95 \pm 66$ & & $117 \pm 77$ & & $114 \pm 77$ & $121 \pm 92$ & $88 \pm 37$ & $92 \pm 40$ \\
\hline $\mathrm{E}_{2}(\mathrm{pmol} / \mathrm{L}) \dagger$ & $224 \pm 103$ & & $187 \pm 128$ & & $238 \pm 114$ & $356 \pm 165$ & $209 \pm 150$ & $150 \pm 66$ \\
\hline $\mathrm{P}(\mathrm{nmol} / \mathrm{L}) \dagger$ & $8.6 \pm 12.44$ & & $6.0 \pm 8.9$ & & $3.8 \pm 4.1$ & $14.6 \pm 12^{c}$ & $4.1 \pm 5.4$ & $5.1 \pm 6.4$ \\
\hline $\mathrm{T}(\mathrm{nmol} / \mathrm{L})$ & $1.04 \pm 0.31^{A}$ & & $1.25 \pm 0.41$ & & $1.06 \pm 0.38$ & $1.26 \pm 0.38$ & $1.07 \pm 0.31$ & $1.18 \pm 0.45$ \\
\hline $\mathrm{A}(\mathrm{nmol} / \mathrm{L})$ & $5.40 \pm 1.73^{A}$ & & $6.27 \pm 1.98$ & & $5.51 \pm 2.25$ & $6.34 \pm 2.49$ & $5.79 \pm 2.08$ & $7.04 \pm 2.18$ \\
\hline DHEAS $(\mu \mathrm{mol} / \mathrm{L})$ & $5.69 \pm 2.17$ & & $5.97 \pm 2.8$ & & $5.16 \pm 2.17$ & $5.86 \pm 3.53$ & $7.22 \pm 2.44$ & $8.09 \pm 2.17$ \\
\hline
\end{tabular}

* Mean ovarian (right + left) \pm SD. Abbreviations as in Table 1.

$\dagger$ Luteal phase.

${ }^{A} p<0.05$, group $1 v s$ Group 2.

${ }^{B} p<0.02$, group 1 vs Group 2.

${ }^{a} p<0.05$, increased first $v s$ last.

${ }^{b} p<0.001$, increased first $v s$ last.

${ }^{c} p<0.005$, increased first $v s$ last.

${ }^{d} p<0.05$, normalized first $v s$ last.

${ }^{e} p<0.001$, normalized first $v s$ last.

values of the first examination and those of the adolescents with regular cycles. Subjects exceeding the normal range were $40 \%(+12 \%)$ for $\mathrm{LH}, 36 \%(+14 \%)$ for testosterone, and $48 \%$ $(+15 \%)$ for androstenedione.

Ovulatory cycles (Table 1 ) increased with respect to the first examination and were $68 \%(+21 \%)$, and mean $\mathrm{E}_{2}$ and progesterone premenstrual levels increased, reaching normal adolescent control values (Table 1). Cycle length decreased (Table 1); 35 subjects (47\%) out of 74 had a normalized menstrual rhythm, and mean height was increased, whereas no changes were registered in mean weight (Table 1).

The hirsutism score did not significantly change, and none of the subjects developed excessive hair growth.

Longitudinal control of the ovaries and correlated data in group 1 and group 2. Fourteen subjects (30\%) out of 46 with normal ovarian volume at the first examination showed an ovarian enlargement at the last examination (Table 2, Fig. 1) exceeding the normal range. In changing ovarian volume, six subjects also changed the homogeneous structure into polycystic structure and one into multifollicular structure. Four multifollicular ovaries became polycystic, whereas three subjects maintained the polycystic structure at the last examination. In the subjects who did not register any significant increase of ovarian volume, changes in the ovarian structure were also rare.

Mean basal endocrine parameters of the 14 subjects did not differ from mean values of group 1 (Table 2). LH and androgen levels slightly increased even though differences were not significant and became similar to those of group 2. A higher percentage of ovulatory cycles with a normalization of cycle length was observed, however, with a significant increase of $E_{2}$ and progesterone levels in the luteal phase.

Six subjects (22\%), out of the 27 with enlarged ovaries at the first examination, showed ovaries within the normal range in the last examination (Table 2, Fig. 2), whereas ovarian structure did not substantially change; three subjects maintained 


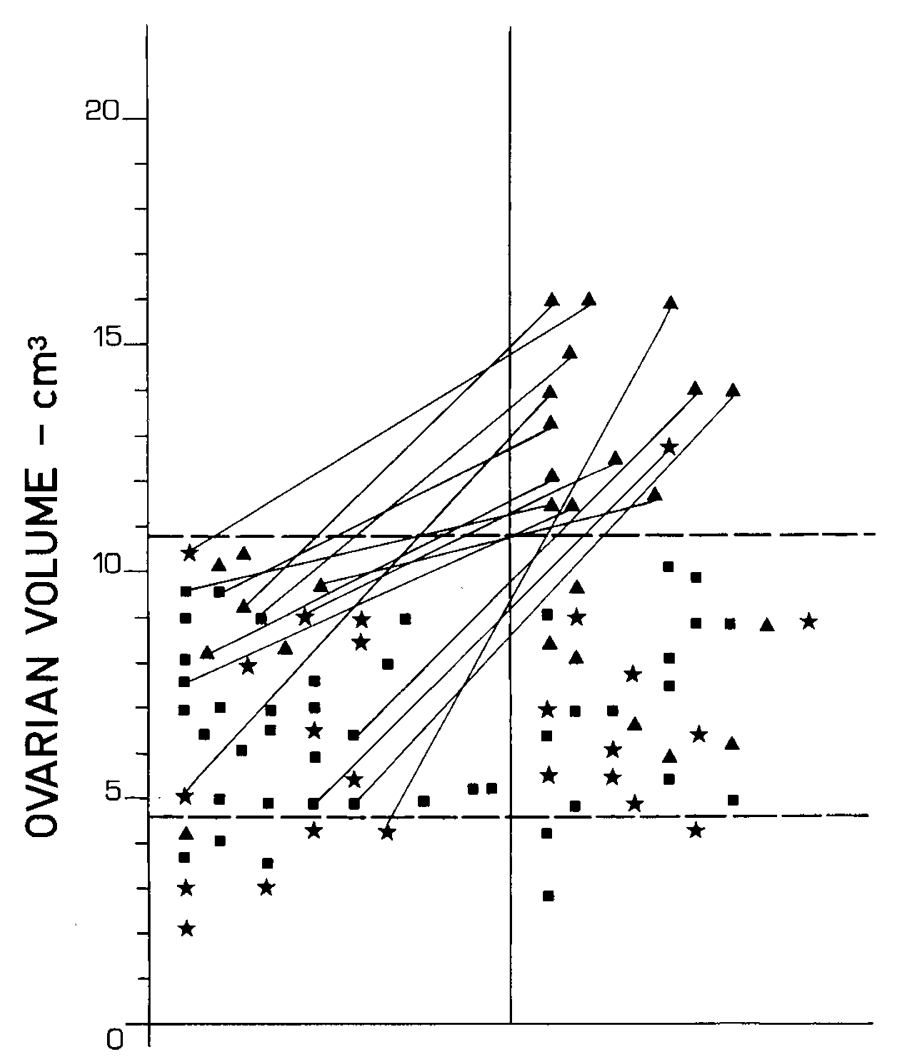

Figure 1. Longitudinal examination of the ovaries. Distribution of the subjects $(n=46)$ with normal ovarian volume during the first control (left) and outcome of the ovarian volume and morphology as found during last control (right). During the last control 14 subjects $(30 \%)$ showed ovarian volume exceeding the normal range; polycystic structure was the dominant feature. Continuous lines connect the subjects with normal ovarian volume during the first control and enlarged ovaries in the last control. Dashed lines indicate normal adult range of ovarian volume. (1) Homogeneous ovary, first control, $n=26$; last control, $n=15$. ( $\star$ Multifollicular ovary, first control, $n=13$; last control, $n=11$. (A) Polycystic ovary, first control, $n=7$; last control, $n$ $=20$

polycystic structure, two converted their polycystic structure into the multifollicular structure, and one showed the opposite trend. Seventy-seven percent of subjects maintained at the last examination the increased ovarian volume found at the first examination, and only rarely were changes of structure observed.

Subjects who normalized ovarian volume did not significantly change their endocrine pattern (Table 2), even though an increase of androstenedione levels was observed, and three subjects had normalized LH values. Cycle length decreased, whereas the ovulatory rate did not increase, and progesterone and $\mathrm{E}_{2}$ levels in the luteal phase were unchanged.

\section{DISCUSSION}

The present data state that three basic features of the ovaries can be found in adolescents during the postmenarcheal period: the homogeneous, the multifollicular, and the polycystic. Homogeneous ovaries prevail in regular ovulatory cycles, even though they are also present in irregular cycles, whereas inhomogeneous ovaries (more polycystic than multifollicular) prevail in irregular menstrual cycles. Moreover, the present data

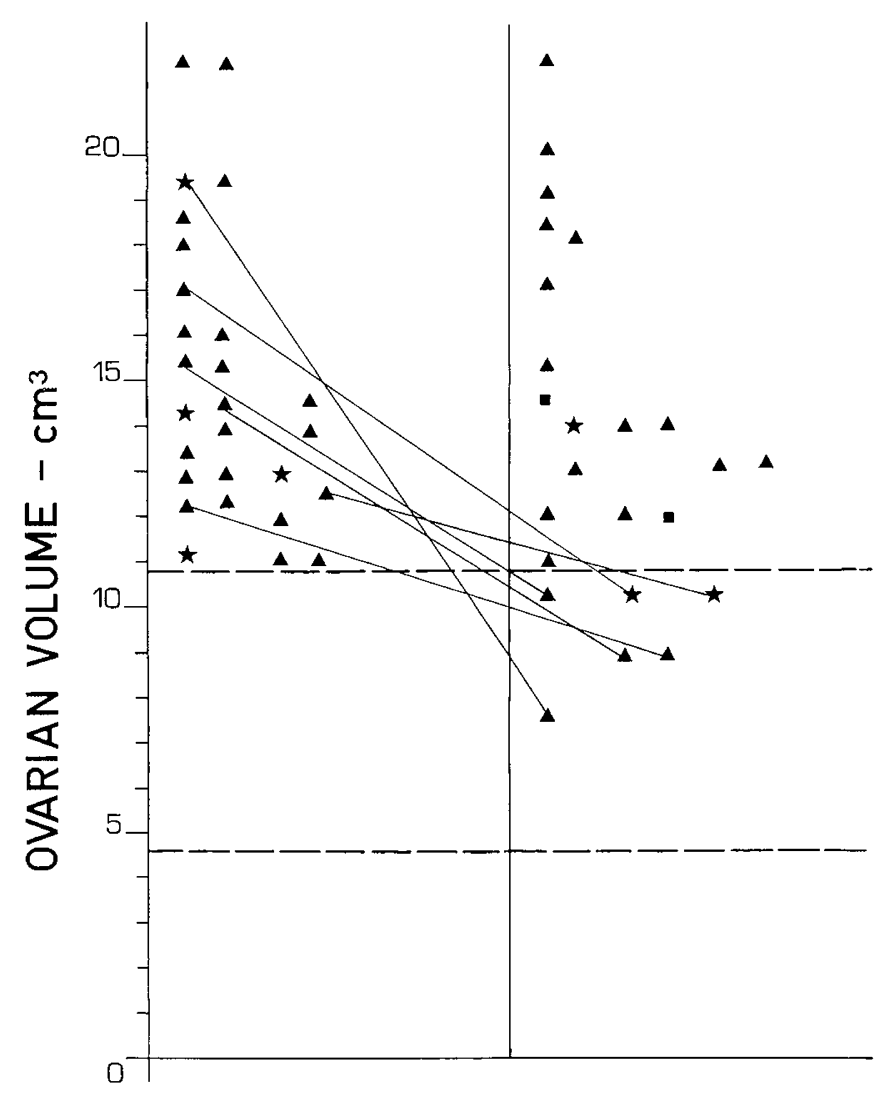

Figure 2. Longitudinal examination of the ovaries. Distribution of the subjects $(n=27)$ who showed the ovarian volume exceeding normal adult range during the first control (left) and outcome of the ovarian volume and morphology during the last control (right). Six (22\%) subjects showed the ovarian volume within the normal range during the last control, but the inhomogeneous structure persisted. Continuous lines connect the subjects with ovaries exceeding normal adult volume during the first control and ovaries within normal range in the last control. Dashed lines indicate normal adult range of ovarian volume. (ם) Homogeneous ovary, first control, $n=0$; last control, $n=2$. ( $\star$ ) Multifollicular ovary, first control, $n=4$; last control, $n=3$. (A) Polycystic ovary, first control, $n=23$; last control, $n=22$.

confirm our previous reports $(3,4)$ which stated that adolescents with irregular cycles tend to have enlarged ovaries compared with adults and adolescents having well established, regular ovulatory cycles. Ovarian structure is linked to ovarian volume in the adolescent period; in fact, polycystic ovaries exhibit the highest volume, and they generally exceed the adult normal range. Homogeneous ovaries show the lowest volume and, together with the multifollicular ones, remain within the normal adult range.

Ovarian structure in the adolescent period is still a controversial matter. In our previous reports $(3,4,8)$ we defined as "multifollicular or multicystic," ovaries with more than four follicles, to stress the contrast between homogeneous structure typical of regular, ovulatory, and mature cycles and inhomogeneous structure, which greatly characterizes adolescents with irregular cycles and a still immature reproductive system. However, we repeatedly referred to them $(3,4,8,10)$ as enlarged and very similar to those found in adult PCOS. We indeed confirm that the ovaries of the irregular menstrual cycles in the adolescent period are frequently and really polycystic as seen by ultrasound examination. The present data 
bring to mind Grinsted and Peter's (11) and Merrill's (12) histologic data emphasizing the frequent and striking similarities between pubertal and adolescent ovaries and those seen in PCOS. Our data also support the preliminary observations of Brook et al. (13) and, more recently, the data of Bridges et al. (2) that referred to the presence of both polycystic and/or multifollicular ovaries in the adolescent period.

The crux of the matter is the ever present difficulty in definition, keeping in mind that 1) the inhomogeneous structure of the ovaries is surprisingly frequent in the adolescent period, 2) it sometimes disappears, and 3) it frequently is not associated with other pathologic signs. We also know that polycystic ovaries may occur in almost a quarter of normal adults without apparent pathologic implications, and not all women with a polycystic structure have problems of infertility (14). Also the multifollicular structure is suggestive of an immature and still developing reproductive system, even though it may often be indicative of a pathologic condition, such as anorexia $(9,15,16)$, or it may evolve, as previously suggested by Stanhope et al. (17), into a polycystic pattern.

The present study is the first longitudinal investigation that documents the outcome of the adolescent ovaries. We emphasize that enlarged and polycystic ovaries become a permanent clinical feature for the majority of the adolescents having irregular cycles without signs of hyperandrogenism, as we previously hypothesized in our cross-sectional studies (8). Moreover, during the postmenarcheal period, volume and polycystic structure of the ovaries tended to increase, whereas homogeneous ovaries and to a lesser extent multifollicular ovaries tended to decrease

Unlike girls with small ovaries, subjects with enlarged and polycystic ovaries had later menarche, and they frequently had higher androgen and LH levels with a lower percentage of ovulatory cycles (Table 2). This study documents the persistence of one or more of these clinical PCOS-like characteristics that are frequently strongly linked (18). This condition has important implications on the adult reproductive function.

The follow-up of cycle length and ovulatory rate documented an increase in the number of regular and ovulatory cycles, despite the persistence of hyperandrogenemia, high $\mathrm{LH}$ levels, and enlarged polycystic ovaries (Table 1). These apparently contradictory data constitute the adolescent background which partly explains Polson et al.'s data (14) showing a high incidence of polycystic structure in an apparently normal randomized adult population and the not infrequent appearance of ovulatory follicles within a polycystic structure (19).

The present report tallies with Apter and Vihko's (20) observations which demonstrated that the endocrine characteristics, formed during puberty and particularly those androgenic, persist at least until $30 \mathrm{y}$ of age and that higher serum androgen concentrations are associated with lower fertility.

Because, during puberty, a high follicular turnover does exist with a progressive increase in the number and size of follicle and in the amount of stroma derived from follicular atresia (21), the sooner the dominant follicle growth takes over, the quicker the ovary will assume a normal adult aspect. The duration of anovulation is probably the key factor responsible for the onset of the persistence and worsening of the polycystic structure (22).

During the period of the study, namely the years after menarche, the ovarian volume changed from normal to enlarged in $30 \%$ of the adolescent population. The ovarian volume exceeded the adult range and the ovarian structure changed from homogeneous or multifollicular to polycystic (Fig. 1). Thus we emphasize that the postmenarcheal period is frequently the starting point or the first time the polycystic tranformation of the ovaries appears. Even though polycystic appearance of the ovaries may have been reported before puberty (23), either in the absence of gonadotropins (24) or when gonadotropin concentrations are physiologically low (2), persistent abnormal androgens and/or abnormal gonadotropin profiles are involved in this process and should probably be considered negative prognostic factors.

By contrast, in some subjects with enlarged multifollicular and polycystic ovaries (22\%) ovarian volume can become normal (Fig. 2). It is well known that multifollicular morphology of the ovaries may revert to normal when ovulation is achieved (15). On the other hand, the possibility of achieving a normal adult echographic image with a homogeneous structure of the ovaries during the postmenarcheal period was not observed for the polycystic ovaries, confirming the observations of Bridges et al. (2). In two cases only a shift from polycystic to multifollicular appearance was detected; this is sufficient to confirm that the transition from one structure to the other and vice versa may occur (Figs. 1 and 2).

Changes in gonadotropin levels and pulsatility represent an important factor in regulating physiologic and pathologic ovarian function, follicular maturation, and steroidogenesis (25, 26). Normalization of volume and structure of the ovaries may partly correlate to gonadotropin modifications which, in our experience (27), generally consists of an LH pulse frequency and amplitude reduction. By contrast, gonadotropin modifications might induce the increase in volume and the structural changes of the ovaries during the post menarcheal period.

Not all the subjects who normalized ovarian volume showed a change in gonadotropin levels (Table 2) and in androgens levels. Whereas when gonadotropin changes happened, often ovarian parameters (such as cystic structure or amount of stroma) did not change. The same happened to the subjects who had an increase in ovarian volume and changed ovarian structure (Table 2); additional intraovarian and independent still unknown regulatory factors may account for this.

Our study indentified, at the beginning of the investigation, a subset of adolescent population with PCOS-like signs. The aggregation of the signs and the duration of the aggregation were variable and none of these symptoms, in itself, is more predictive than another for further pathologic development or for normalization of the reproductive function.

The longitudinal study documented the persistence in most girls of the PCOS-like parameters discovered at the beginning of the investigation. Among them, the endocrine parameters and particularly LH levels were the most flexible, variable, and susceptible of at least temporarily correction. Unlike these parameters, the ovarian volume and morphology were less 
susceptible of variations and once altered, they persisted in most cases.

\section{REFERENCES}

1. Orsini LF, Salardi S, Pilu G, Bovicelli L, Cacciari E 1984 Pelvic organs in premenarcheal girls: real-time ultrasonography. Radiology 153:113-116

2. Bridges NA, Cooke A, Healy MJR, Hindmarsh PC, Book CGD 1993 Standards for ovarian volume in childhood and puberty. Fertil Steril 60:456-460

3. Venturoli S, Porcu E, Fabbri R, Paradisi R, Orsini LF, Flamigni C 1984 Ovaries and menstrual cycles in adolescence. Gynecol Obstet Invest 17:219-222

4. Venturoli S, Porcu E, Fabbri R, Paradisi R, Ruggeri S, Bolelli G, Orsini LF, Gabbi D, Flamigni C 1986 Menstrual irregularities in adolescents: hormonal pattern and ovarian morphology. Horm Res 24:269-279

5. Stanhope R, Adams J, Jacobs HS, Brook CGD 1985 Ovarian ultrasound assessment in normal children, idiopathic precocious puberty and during low dose pulsatile GnRH therapy of hypogonadotrophic hypogonadism. Arch Dis Child 60:116-119

6. Apter D, Viinikka L, Vihko R 1978 Hormonal pattern of adolescent menstrual cycles. J Clin Endocrinol Metab 47:944-954

7. Venturoli S, Porcu E, Fabbri R, Paradisi R, Magrini O, Orsini LF, Ruggeri S 1985 Endocrine profiles and ovarian aspects in adolescent menstrual abnormalities. In Flamigni C, Venturoli S, Givens JR (eds) Adolescence in Females. Year Book, Chicago, pp 239-250

8. Venturoli S, Porcu E, Fabbri R, Magrini O, Paradisi R, Pallotti G, Gammi L, Flamigni C 1987 Postmenarcheal evolution of endocrine pattern and ovarian aspects in adolescents with menstrual irregularities. Fertil Steril 48:78-85

9. Adams J, Polson DW, Abdulwahid N, Morris DV, Franks S, Mason HD, Tucker M, Prince J, Jacobs HS 1985 Multifollicular ovaries: clinical and endocrine features and response to pulsatile gonadotropin releasing hormone. Lancet 2:1375-1378

10. Venturoli S, Porcu E, Fabbri R 1989 Polycystic ovarian syndrome and adolescence. In Genazzani AR, Petraglia F, Volpe A (eds) Progress in Gynecology and Obstetrics. Parthenon, Carnforth, UK, pp 539-544

11. Grinsted J, Peters H 1979 The human ovary during puberty. Ann Biol Anim Biochim Biophys 19:1455-1460

12. Merrill JA 1963 The morphology of the prepubertal ovary: relationship to the polycystic ovary syndrome. South Med J 56:225-231

13. Brook CGD, Jacobs HS, Stanhope R 1988 Polycystic ovaries in childhood. BMJ 296:878
14. Polson DW, Wadsworth J, Adams J, Franks S 1988 Polycystic ovaries a common finding in normal women. Lancet 1:870-872

15. Treasure JL, King EA, Gordon PAL, Wheeler M 38 Russel GFM 1985 Cystic ovaries: a phase of anorexia nervosa. Lancet 2:1379-1383

16. Futterweit W, Yeh HC, Mechanick JI 1988 Ultrasonographic study of ovaries of 19 women with weight loss-related hypothalamic oligo-amenorrhea. Biomed Pharmacother 42:279-284

17. Stanhope R, Adams J, Brook CGD 1988 Evolution of polycystic ovaries in a girl with delayed menarche. A case report. J Reprod Med 33:482-484

18. Venturoli S, Porcu E, Fabbri R, Paradisi R, Gammi L, Passarini M, Orsini LF, Flamigni C 1986 Ovarian multifollicularity, high LH and androgen plasma levels, and anovulation are frequent and strongly linked in adolescent irregular cycles. Acta Endocrinol 111:368-372

19. Adams J, Polson DW, Franks S 1986 Prevalence of polycystic ovaries in women with anovulation and idiopathic hirsutism. BMJ 293:355-359

20. Apter D, Vihko R 1989 Endocrine determinants of fertility: serum androgen concentrations during follow-up of adolescents into the third decade of life. J Clin Endocrinol Metab 71:970-974

21. Peters H 1979 The human ovary in childhood and early maturity. Eur J Obstet Gynecol Reprod Biol 9:137-144

22. Venturoli S, Fabbri R, Porcu E, Paradisi R, Orsini LF, Brondelli L, Ruggeri S, Flamigni C 1989 Endocrine and ovarian parameters at various frequencies of ovulation in adolescents. Arch Gynecol Obstet 246:107-114

23. Rao JK, Chihal HJ, Johnson CM 1985 Primary polycystic ovary syndrome in a premenarcheal girl: a case report. J Reprod Med 30:361-366

24. Stanhope R, Adams J, Pringle JP, Jacobs HS, Brook CGD 1987 The evolution of polycystic ovaries in a girl with hypogonadotropic hypogonadism before puberty and during puberty induced with pulsatile gonadotropin-releasing hormone. Fertil Steril 47:872-875

25. Venturoli S, Porcu E, Fabbri R, Magrini O, Gammi L, Paradisi R, Focacci M, Bolzani R, Flamigni C 1988 Episodic pulsatile secretion of FSH, LH, prolactin, oestradiol, oestrone, and LH circadian variations in polycystic ovary syndrome. Clin Endocrinol 28:93-107

26. Venturoli S, Porcu E, Gammi L, Magrini O, Fabbri R, Paradisi R, Flamigni C 1987 Different gonadotropin pulsatile fashions in anovulatory cycles of young girls indicate different maturational pathways in adolescence. J Clin Endocrinol Metab 65:785-791

27. Venturoli S, Porcu E, Fabbri R, Magrini O, Gammi L, Paradisi R, Flamigni C 1992 Longitudinal evaluation of the different gonadotropin pulsatile patterns in anovulatory cycles of young girls. J Clin Endocrinol Metab 74:836-841 